\title{
Inovação pedagógica universitária mediada pelo Facebook e YouTube: uma experiência de ensino-aprendizagem direcionado à geração-Z
}

\section{University-Level pedagogical innovation mediated by Facebook and YouTube: a teaching-learning experience directed at the Z-generation}

\author{
Luiz Fernando Quintanilha*
}

\begin{abstract}
RESUMO
A educação superior tem encontrado grandes desafios em função das velozes transformações da sociedade e, neste sentido, a aplicação de métodos pedagógicos inovadores que estimulem os alunos a criar e a desenvolver o espírito científico e reflexivo é constantemente requisitada. Entretanto, primeiramente, deve-se considerar a utilização de estratégias de aproximação com os estudantes, especialmente com os mais jovens, nascidos a partir de meados da década de 1990, que estão, atualmente, ingressando nas universidades. Para este grupo de estudantes, denominado de Geração Z, é essencial que os educadores renovem seus métodos de ensino para melhorar o processo de aprendizagem. Neste estudo, é avaliado se a utilização de tecnologias virtuais como Facebook e YouTube apresenta bons resultados no processo de ensino-aprendizagem de estudantes de uma universidade de Salvador. O Facebook foi utilizado como principal canal de comunicação entre professor e alunos; o YouTube, como um canal para compartilhamento de videoaulas desenvolvidas pelos próprios estudantes. Ambas as propostas foram sugeridas e não impostas aos estudantes. Ao final foi aplicado um
\end{abstract}

DOI: $10.1590 / 0104-4060.50027$

* Universidade Salvador. Escola Ciências da Saúde. Salvador, Bahia, Brasil. Campus Professor Barros. Av. Luís Viana Filho, no 3146. Paralela. CEP. 41.720-200. E-mail: luiz. mesquita@pro.unifacs.br 
questionário semiestruturado para avaliar a percepção discente. Verificou-se um alto grau de participação dos estudantes nas atividades propostas. Além disso, a percepção deles de que tais atividades foram eficazes para a construção do conhecimento foi o principal indicador positivo da análise, o que permitiu inferir que o uso dessas tecnologias é eficaz no processo de aprendizado dos estudantes, especialmente dos mais jovens.

Palavras-chave: Facebook. YouTube. Educação. Geração Z.

\begin{abstract}
Higher education has been facing great challenges due to rapidly occurring transformations in society. In this context, the application of innovative teaching methods that encourage students to create and develop scientific and critical thinking is constantly required. Nevertheless, one should first consider the use of strategies for approaching students - especially those from a younger generation, born in the mid-90s, who are currently initiating college. For this group of students, collectively referred to as Generation Z, it is essential that educators renew their teaching methods to improve the learning process. In this study, the use of virtual tools such as Facebook and YouTube was evaluated to determine their effect in higher-education student learning at the University of Salvador, Bahia. Facebook was used as the main communication tool between professors and students, and YouTube was used as a channel for virtual lessons developed by the students themselves. Both proposals have been suggested to but not demanded from the students. In the end, a semi-structured questionnaire was applied to assess student perception. As a result, we observed a high degree of student participation in the methodologies proposed. Moreover, their perception that the methods were effective for knowledge acquisition was the main positive indicator of the analysis. In conclusion, the use of virtual tools can be an effective method in the learning process of students, especially where younger ones are concerned.
\end{abstract}

Keywords: Facebook. YouTube. Education. Generation Z.

\title{
Introdução
}

Atualmente, o ensino superior tem encontrado grandes desafios em função das velozes e profundas transformações da sociedade e as necessidades do mercado de trabalho. Na construção dos currículos universitários, são sugeridos modelos de ensino que englobem inovações em educação (CORTELLA, 2014; 
MITRE et al., 2008; MORÁN, 2015; BRASIL, 1996; 2001). Em consonância, espera-se que a relação educador-estudante se renove para o acompanhamento deste ritmo acelerado de mudanças sociais e comportamentais.

Um dos benefícios desses modelos é o maior envolvimento e satisfação do estudante com a disciplina/curso com maior e, consequentemente, melhor aprendizado. A aprendizagem que envolve a autoiniciativa e alcança dimensões afetivas e intelectuais torna-se mais duradoura e sólida. Para que este modelo funcione, o professor deve servir como tutor/moderador/provocador das atividades desenvolvidas pelos próprios estudantes. Para que isso seja feito de maneira efetiva, é desejável que o professor esteja próximo do cotidiano do alunado e não apenas seja o ator principal de um único encontro semanal durante alguns poucos meses. Propõe-se, portanto, a substituição do modelo tradicional de aulas expositivas para outro modelo que alcance os objetivos pedagógicos que são almejados na universidade (MASETTO, 2004).

É coerente pensar que algumas instituições de ensino superior (IES), em função das dificuldades de promover mudanças mais profundas nas práticas de ensino - que passam desde reestruturações nos espaços de aula até a aplicação de avaliações individualizadas, tendem a optar pela inserção de métodos mais inovadores, os quais aproximam o aluno da instituição, mantendo-o interessado, participativo e motivado, para possibilitar, finalmente, um melhor aprendizado. Da mesma forma, muitos professores relatam grandes dificuldades na adaptação para os novos modelos pedagógicos que exigem, muitas vezes, inúmeras quebras de paradigmas.

Muitas são as propostas para uma readequação no processo de ensino-aprendizagem e na relação professor-aluno (MORÁN, 2015), no entanto, na verdade, boa parte delas, para a grande maioria dos professores e IES, ou são utópicas em curto prazo, ou são extremamente difíceis de serem realizadas pelos mais diversos motivos. Portanto pequenos passos podem ser de grande valia para a melhora na qualidade do ensino.

Nesse sentido, para que essa renovação proposta seja possível, mesmo em níveis sutis, é imprescindível, por parte dos professores, a adequação a esta nova realidade a qual se difere bastante da que se viveu outrora e isto, é claro, exige tempo, trabalho e dedicação (MASETTO, 2004). Portanto, como consequência dessa mudança de atitude, espera-se a formação de melhores profissionais e cidadãos oriundos da universidade.

A motivação para o desenvolvimento deste trabalho surgiu a partir da observação de que as novas turmas universitárias são amplamente compostas por indivíduos nascidos em meados da década de 1990, os quais integram a chamada Geração Z (MCCRINDLE, 2014). Esta geração que nasceu e cresceu em uma sociedade com a possibilidade de acesso à internet, computadores, celulares, am- 
bientes virtuais e jogos é objeto de estudo de diversos segmentos e não poderia ser diferente no ramo da Educação (JONES; JO; MARTIN, 2007). O ato de lecionar para uma sala repleta dos estudantes é, frequentemente, reportado como um dos maiores desafios atuais por boa parte dos professores, pois os métodos de ensino tradicionais têm extrema dificuldade em envolver indivíduos com as características da Geração Z, o que dificulta imensamente o processo de aprendizagem e a relação professor-aluno. Esses estudantes têm acesso simples e extremamente rápido à informação - não necessariamente de boa qualidade - e dificuldade em se manterem atentos e focados em uma única atividade por longos períodos.

Dados apontam para uma utilização alta da internet pelo brasileiro, especialmente por jovens e adultos (CIRIBELI; PAIVA, 2011). Nesse sentido, um levantamento recente do Instituto Brasileiro de Geografia e Estatística (IBGE) revela que a maioria dos cursos universitários é composta, majoritariamente, por jovens alunos, os quais utilizam computadores, tablets, smartphones na sua vida cotidiana, inclusive para fins educacionais (PORTAL BRASIL, 2016). Em atividades realizadas em sala de aula, mesmo com a oferta de livros didáticos, é extremamente comum notar que a maioria dos alunos busca as informações necessárias por meio desses itens, o que não deve ser desmotivado, mas, sim, orientado.

Com isso, é lógico pensar que a utilização dessas mesmas tecnologias por parte dos professores é bem-vinda, especialmente quando direcionadas especificamente ao corpo discente em questão. Quanto mais informações com finalidades educacionais estiverem disponíveis aos alunos, maiores as chances deles as acessarem, familiarizando-se com elas e tornando-as parte de uma rotina de estudos.

São exemplos notórios dessas tecnologias supracitadas as interfaces digitais. Redes sociais e canais virtuais de vídeos são extensamente utilizados pela população, inclusive a universitária, o que dá ao professor possibilidades extras de comunicação com o aluno. O compartilhamento de informações e videoaulas é de acesso fácil e atrativo para a maioria dos estudantes e estas podem ser ferramentas poderosas no processo de ensino-aprendizagem.

O Facebook é a rede social mais famosa do mundo, com mais de 1,5 bilhão de usuários (THE STATISTICS PORTAL, 2016a). No Brasil, em 2016, este número está estimado em 90 milhões de pessoas (THE STATISTICS PORTAL, 2016b). Essa plataforma possui uma série de ferramentas interessantes, boa parte das vezes subutilizadas, que podem ser instrumentos muito úteis para o processo de ensino-aprendizagem (CASTILHO et al., 2014; FERREIRA; CORRÊA; TORRES, 2012). Por sua vez, o YouTube é o mais famoso site de compartilhamento de vídeos do mundo contando com mais de 1 bilhão de usuários que geram bilhões de visualizações de vídeos todos os dias (YOUTUBE, 2016). 
As potencialidades de aplicação desses dois exemplos no ensino têm sido recentemente reportadas e, portanto, já não se pode ignorar a força dessas plataformas no processo educacional, incluindo o ensino superior (BOSCH, 2009; BOTTENTUIT JUNIOR; COUTINHO, 2009). É importante que os professores que lidam com esses jovens alunos estejam cientes desse novo comportamento e abertos a adaptações ao novo estilo de vida deles, incluindo o comportamento educacional. A utilização desses websites como aliados no processo pedagógico gera proximidade com o estudante e pode gerar resultados excelentes $\mathrm{BOSCH}$, 2009; MORAN; SEAMAN; TINTI-KANE, 2011). Corroborando o supracitado, segundo Souza e Schneider (2012), ao elaborar propostas de ensino que permitam a utilização das tecnologias on-line de forma pedagógica, o docente estará promovendo maior autonomia do aluno.

Outro ponto de destaque na utilização desses websites é a não necessidade de recursos financeiros, o que torna essa estratégia altamente viável para qualquer instituição de ensino e para qualquer professor que queira dar um passo na direção da aproximação com seu alunado. Adicionalmente, a variedade de atividades que estes websites são capazes de produzir é enorme, vão desde compartilhamento de informações, textos e vídeos, até a criação de jogos, questionários, pesquisas e avaliações.

Assim o presente trabalho se propõe a avaliar a utilização do Facebook e YouTube como método acessório no ensino de uma disciplina da área de saúde para dois cursos universitários. O texto apresenta a forma como esses métodos foram aplicados e, fundamentalmente, revela as características do alunado envolvido e a avaliação discente acerca dos métodos aqui propostos.

\section{Objetivo}

O objetivo deste trabalho é avaliar o êxito da inserção de tecnologias virtuais para o processo de ensino-aprendizagem de jovens universitários, bem como apresentar possibilidades de uso e determinar o perfil do alunado em estudo.

\section{Métodos}

O presente trabalho obteve anuência da IES e envolveu estudantes de dois cursos na área de saúde de uma universidade privada de Salvador, capital da Bahia. 
Com base nas informações supracitadas, buscou-se alternativas para que o ensino da disciplina ministrada pelo autor se tornasse mais atrativo para os estudantes. Além disso, as estratégias utilizadas serviram para que os alunos fossem estimulados a criar e desenvolver o espírito científico e reflexivo, o que é preconizado nas leis de diretrizes da educação (BRASIL, 1996).

Para tal, buscou-se a aproximação com alunos de duas maneiras: (a) Criação de um grupo de discussão no Facebook, cuja proposta foi facilitar a troca de informações relativas à disciplina e ao compartilhamento de material de ensino em português e inglês e (b) criação de um canal no YouTube, cuja proposta foi criar um espaço para que os alunos compartilhassem videoaulas e, assim, fosse possível criar uma videoteca virtual contemplando diferentes tópicos no estudo da disciplina. Foi facultada aos alunos a decisão pela adesão ou não a proposta.

A avaliação geral de êxito se deu por meio de um questionário semiestruturado contendo doze questões fechadas (vide seção "Resultados e Discussão"), o qual foi devidamente preenchido pelos estudantes. Os dados quantitativos oriundos desta coleta foram processados e analisados por meio do GraphPad Prism 7. Adicionalmente, outras cinco questões do questionário permitiram a inserção de comentários e críticas em relação aos métodos, originando um feedback mais específico e individualizado do corpo discente e a construção de uma análise qualitativa que é exemplificada durante este texto com fragmentos de respostas dos participantes.

\section{Resultados e discussão}

Após a conclusão do semestre letivo, uma análise das contribuições pedagógicas da utilização das estratégias anteriormente descritas foi realizada. Para tal, um questionário foi aplicado aos estudantes que integraram as turmas nas quais os métodos foram propostos. Houve 88 devolutivas, o que proporcionou a realização de uma análise direta das características dos alunos que participaram, suas impressões, além da visão sobre o impacto das atividades no processo de aprendizagem.

O relato de alguns estudantes permitiu a constatação de que os métodos utilizados foram capazes de tornar a disciplina ministrada mais atrativa.

\section{P - Quais foram suas impressões gerais sobre a disciplina Homeostase, conduzida pelo professor?}


E1 - No começo a matéria parecia ser muito difícil, mas os métodos utilizados pelo professor foram muito importantes para ter uma visão diferente da matéria, uma visão boa da matéria. A disciplina foi interessante e divertida de aprender.

Inicialmente, observou-se uma alta taxa de permanência de alunos veteranos, ou seja, aqueles sujeitos que já haviam passado pela disciplina no grupo de discussão do Facebook. Essa observação é interessante pelo fato de a permanência no grupo ser inteiramente opcional e por contribuir para o enriquecimento da discussão (SCHLEMMER, 2015). Além disso, houve um crescente número de solicitações de participação, incluindo os estudantes de outros semestres nos quais a disciplina em questão sequer é oferecida, além de alunos de outras instituições de ensino da cidade. Nesses dois últimos casos, as solicitações foram negadas por não estarem de acordo com os objetivos da criação do grupo. Finalmente, houve uma considerável participação de alunos no compartilhamento e utilização de materiais educacionais. A análise do conjunto dessas observações nos permite inferir que a utilização de grupos em redes sociais pode ser uma boa alternativa para a troca de informações entre professores e estudantes.

$P$ - Qual sua visão sobre o uso das redes sociais no processo de aprendizagem da disciplina?

E2 - O professor sempre manteve uma boa relação com os alunos disponibilizando [...] ferramenta como o Facebook para chegar mais próximo dos alunos.

E3 - Acredito ser importante, pois se encaixa na realidade que vivemos. É uma forma mais dinâmica de aprender e compartilhar os assuntos.

E4 - É maravilhoso, não que as ferramentas utilizadas pela instituição não sejam boas, mas com o Facebook a aproximação com o aluno fica muito mais fácil, pela agilidade de conexão já que estamos online $24 \mathrm{~h}$.

Em relação ao YouTube, a criação de um canal foi proposta para que os alunos desenvolvessem materiais audiovisuais sobre algum aspecto de interesse abordado na disciplina.

O intuito foi desafiá-los e fazer com que os estudantes colocassem em prática seus conhecimentos e elaborassem videoaulas nas quais sua criatividade 
e habilidades com programas de computador fossem parte da construção do conhecimento. Por se tratar de uma atividade não obrigatória, a constatação de uma participação de mais de $70 \%$ de todo o corpo discente se mostrou significativa (Gráfico 1). Essa porcentagem foi, na realidade, ainda maior, pois, por limitação metodológica, não foi possível excluir os alunos matriculados, mas não assíduos, do número total de alunos. É possível que a liberdade de escolha do aspecto da disciplina que mais os agradaram e o estímulo para a elaboração de uma atividade diferente das convencionais tenham sido aspectos determinantes para a grande aderência dos alunos à atividade proposta.

\section{GRÁFICO 1 - VALORES ABSOLUTOS E PERCENTUAIS DE ALUNOS MA- TRICULADOS NA DISCIPLINA QUE PARTICIPARAM DA ELABORAÇÃO DE VIDEOAULAS}

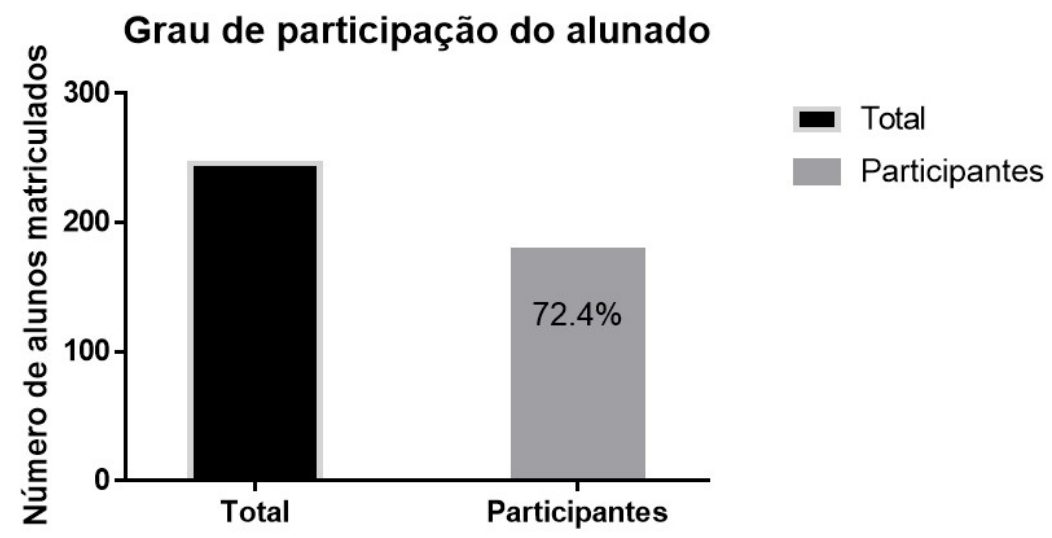

FONTE: Elaborado pelo autor.

Na parte discursiva do questionário, constatou-se uma série de elogios às estratégias adotadas. Foram realizados comentários sobre a importância das atividades na construção do conhecimento que os permitiram ter um maior grau de entendimento em disciplinas mais avançadas.

P - Sobre a elaboração de um vídeo para o Youtube, foi a primeira vez que você elaborou um vídeo para esse propósito? Você acha que isto foi significativo para o seu aprendizado? Por quê?

E5 - Já realizei vídeos de trabalhos escolares e de faculdade, contudo para o youtube foi a primeira vez e acho válido para um número maior 
de pessoas terem acesso a informação e não ficar restrita apenas ao ambiente e pessoas da sala de aula. Para elaboração de um vídeo, é preciso dedicar um tempo para discutir o assunto, buscar informações que possam ser criativas e importantes de serem passadas, implicando em um domínio do conteúdo. Foi uma experiência significativa sim e o conhecimento adquirido ajudou muito em disciplinas posteriores.

E6 - Foi a primeira que vez que elaborei um trabalho escolar para o youtube. [...] por causa desse vídeo tive que buscar várias informações em outros livros de diversas disciplinas, referência em artigos internacionais e essa busca pelo conhecimento para trazer um vídeo de qualidade foi uma experiência única para mim e para meus companheiros de equipe.

Corroborando o que foi previamente citado em relação a Geração Z e a utilização da internet para o processo de aproximação e aprendizagem, o questionário revelou que $100 \%$ dos estudantes avaliados utilizam internet e, destes, apenas $1,1 \%$ a utiliza com uma frequência considerada baixa (Gráfico 2).

\section{GRÁFICO 2 - FREQUÊNCIA DE UTILIZAÇÃO DA INTERNET PELOS ES- TUDANTES}

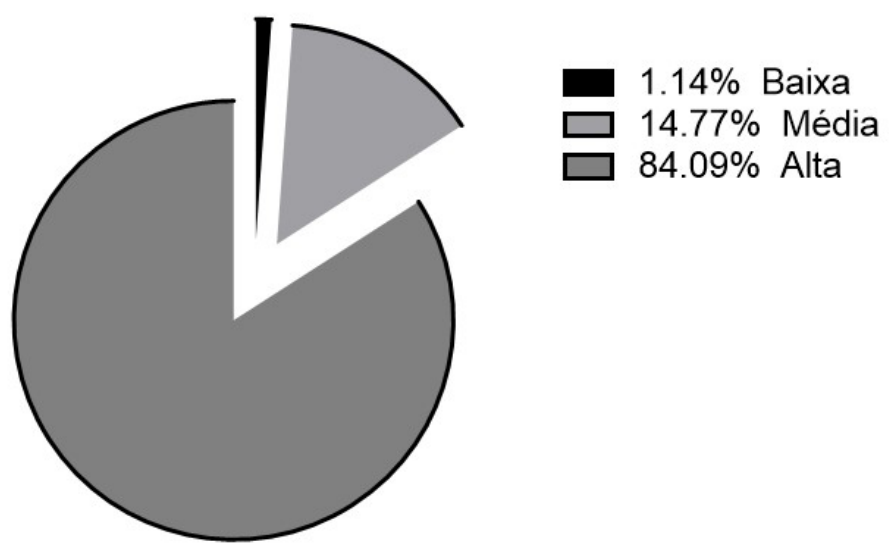

Total $=88$

FONTE: Elaborado pelo autor.

NOTA: A frequência alta significa "diariamente", segundo o questionário. 
Em relação à utilização de redes sociais, $90,9 \%$ dos estudantes têm conta no Facebook (Gráfico 3A) com uma frequência de acesso majoritariamente alta (Gráfico 3B). Esses números revelam o impacto que a utilização de redes sociais no processo de aprendizagem pode ter nesse grupo de estudantes. Apesar de o trabalho ter sido desenvolvido em uma IES privada, acredita-se que os mesmos resultados observados aqui poderiam ser replicados em quaisquer outras universidades devido ao crescente acesso dos brasileiros aos aparelhos com internet (IBGE, 2014).

Ainda em relação à utilização de redes sociais, cerca de $88,8 \%$ dos alunos entrevistados com acesso ao Facebook participaram/participam do grupo de discussão da disciplina (Gráfico 3C) e 78,4\% deles acessaram/acessam o grupo (Gráfico 3D). Tais acessos tiveram motivações distintas e o Gráfico 3E demonstra a distribuição heterogênea dos principais objetivos dos acessos.

Um dado interessante obtido desse questionário foi a opinião dos estudantes acerca da existência do grupo no Facebook: Cerca de 97\% dos alunos julgam interessante a existência do grupo, incluindo aqueles que não o acessam com frequência (Gráfico 3F). Esse resultado revela o quão presente e importante as redes sociais estão na rotina dos estudantes e quão receptivos esses alunos estão para a ocupação desse espaço pelo professor, pela disciplina lecionada, pela informação correta e profunda. A utilização deste ambiente é de grande valia para a transmissão de conhecimento, já que todos esses elementos supracitados estarão, constantemente, presentes no cotidiano e não ficarão restritos à sala de aula em dias específicos.

Outra tecnologia utilizada na disciplina é o website de vídeos YouTube. O questionário revelou que um número ainda maior de estudantes o utiliza (98,9\%; Gráfico 4A) com uma frequência de acesso média ou alta para cerca de $80 \%$ deles (Gráfico 4B); apenas um estudante avaliado relatou não utilizar o site em questão. Em conjunto, essas informações sugerem que o YouTube pode ser potencialmente explorado no processo de aprendizagem.

Nessa perspectiva, um trabalho extra de caráter facultativo foi proposto na disciplina ministrada. Este trabalho baseou-se na elaboração de arquivos audiovisuais de até 15 minutos de duração para compartilhamento via canal da disciplina, gerando uma videoteca virtual. Como anteriormente citado, o índice de participação foi alto (mais de 70\%; Gráfico 1). Mais interessante ainda foi verificar que $95 \%$ dos estudantes que realizaram esta atividade opcional consideraram que ela contribuiu significativamente para o aprendizado (Gráfico 4C), o que foi, de fato, o principal objetivo da proposta. 


\section{GRÁFICO 3 - INSERÇÃO DO FACEBOOK NO PROCESSO DE ENSINO- -APRENDIZAGEM}

A

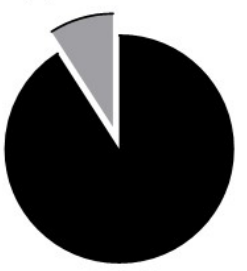

Total $=88$

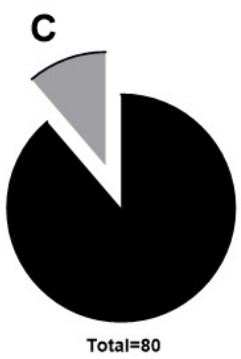

E

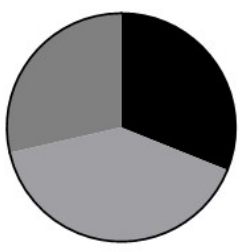

Total=119
B

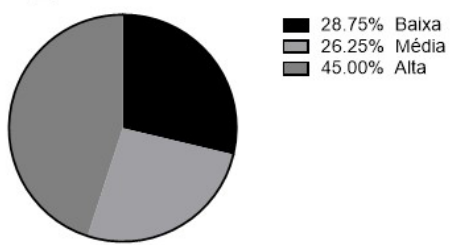

Total $=80$

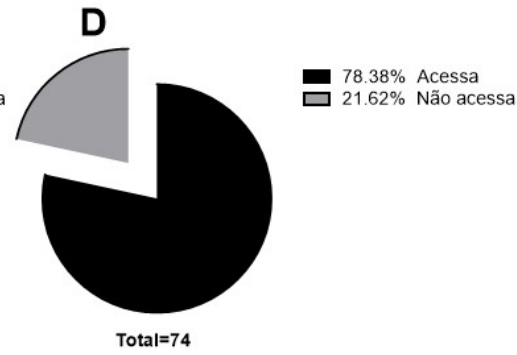

$\mathbf{F}$

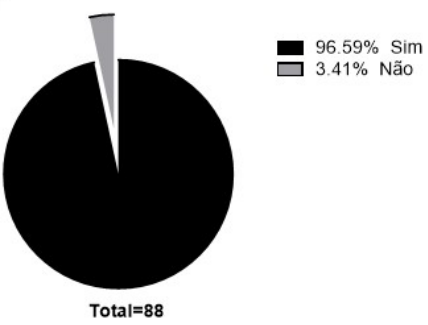

FONTE: Elaborado pelo autor. 


\section{GRÁFICO 4 - INSERÇÃO DO YOUTUBE NO PROCESSO DE ENSINO- APRENDIZAGEM}

A

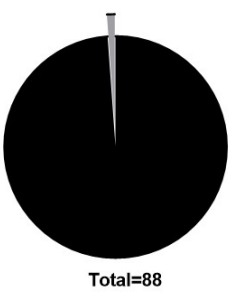

C

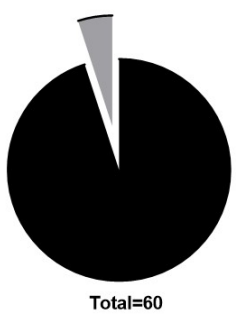

$98.86 \% \mathrm{Sim}$ $\square 1.14 \%$ Não
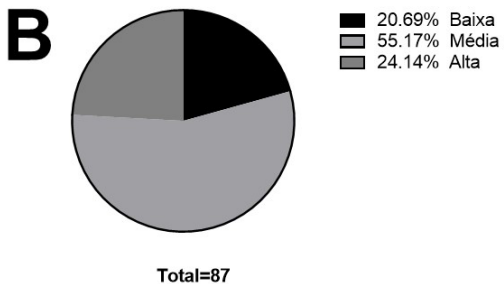

$95.00 \% \mathrm{Sim}$
$5.00 \%$ Não

FONTE: Elaborado pelo autor.

Até a data de submissão deste trabalho, os vídeos inicialmente selecionados para fazer parte desta videoteca possuíam mais de 4 mil visualizações, incluindo, ainda que em proporções menores, acessos oriundos de países como Portugal, Itália, Argentina e Estados Unidos. Esses números são significativos, especialmente em função da população envolvida na sua produção (turmas de primeiro semestre de dois cursos de uma única universidade de Salvador, Bahia). Esses números sugerem fortemente a força da internet e o quanto um trabalho realizado exclusivamente por estudantes pode alcançar.

Nota-se, pelos dados aqui apresentados, que websites como Youtube e $\mathrm{Fa}$ cebook podem ser eficazmente utilizados para a prática docente. Desde que haja um objetivo claro, esses espaços podem servir como uma modalidade didática acessória cumprindo um papel interessante no processo de aprendizagem dos estudantes. Quando o discente participa, colabora, compartilha, curte, ele está inserido e é ator de uma rede social de seu próprio cotidiano, cujo principal objetivo é o aprendizado. Quando este mesmo estudante pensa, reflete, elabora e divulga um arquivo audiovisual acerca de um tema referente à disciplina, ele troca de papel com o professor, exercendo autonomia na elaboração de um projeto e na criação de um produto que certamente contribui para a incorporação de um novo aprendizado ou aprofundamento de um já existente. 
Não há o que temer em relação a aplicação de métodos que envolvam tecnologias virtuais, pois os alunos da geração $\mathrm{Z}$ estão completamente familiarizados com elas. Aqui apresentamos apenas duas ferramentas úteis no processo de ensino-aprendizagem, mas existem diversos outros websites que podem ser utilizados com propósitos semelhantes. Isso certamente exige um esforço docente permanente no sentido de se apropriar dessas tecnologias e pensar em meios didáticos eficazes de utilização e na aceitação delas pelos alunos (BERBEL, 2011; MOREIRA; MONTEIRO, 2015). As implicações pedagógicas que essas ações podem ter são promissoras e devem ser melhor exploradas, documentadas e divulgadas para superarmos os desafios impostos pela educação do século XXI.

\section{Conclusão}

Há, portanto, um claro conjunto de evidências quantitativas e qualitativas de que a aplicação dos métodos aqui descritos obteve êxito em seu objetivo. Com alta taxa de participação e feedback positivo, os alunos demonstraram interesse e envolvimento. Dessa forma, recomenda-se que professores atentem para a mudança de comportamento dos jovens alunos da Geração Z e proponham estratégias inovadoras que envolvam a internet, especialmente aqueles websites nos quais a maioria dos alunos tem claro interesse e habilidade.

\section{Agradecimentos}

Agradeço aos professores Neilton Silva e Rodrigo Sampaio pelas sugestões para a elaboração deste artigo. Também agradeço imensamente aos meus alunos que participaram através do preenchimento do questionário disponibilizado.

\section{REFERÊNCIAS}

BERBEL, N. A. N. As metodologias ativas e a promoção da autonomia de estudantes. Semina: Ciências Sociais e Humanas, v. 32, n. 1, p. 25-40, 2011. 
BOSCH, T. E. Using online social networking for teaching and learning: Facebook use at the University of Cape Town. Communicatio, v. 35, n. 2, p. 185-200, nov. 2009.

BOTTENTUIT JUNIOR, J. B.; COUTINHO, C. P. Desenvolvimento de vídeos educativos com o Windows Movie Maker e o YouTube: uma experiência no Ensino Superior. Universidade Lusófona de Humanidades e Tecnologias, abr. 2009. Disponível em: $<$ http://repositorium.sdum.uminho.pt/handle/1822/9019>. Acesso em: 22 maio. 2016.

BRASIL. Presidência da República. Lei 9.394, de 20 de dezembro de 1996. Estabelece as diretrizes e bases da educação nacional. 1996. Disponível em: <http://www.planalto. gov.br/ccivil_03/leis/L9394.htm>. Acesso em: 21 maio 2016.

BRASIL. Ministério da Saúde/Ministério da Educação. Secretaria de Políticas de Saúde/ Secretaria de Ensino Superior. Projeto de incentivo a mudanças curriculares em cursos de medicina. Brasil, 2001.

CASTILHO, A. M. D. et al. A rede social facebook como ferramenta pedagógica no processo ensino-aprendizagem de Língua Inglesa Revista Transformar. 2014. Disponível em: $<$ http://www.fsj.edu.br/transformar/index.php/transformar/article/view/12>. Acesso em: 22 maio 2016.

CIRIBELI, J. P.; PAIVA, V. H. P. Redes e mídias sociais na internet: realidades e perspectivas de um mundo conectado. Revista Mediação, v. 13, n. 12, 2011.

CORTELLA, M. S. Educação, Escola e Docência. 1. ed. São Paulo: Cortez Editora, 2014. FERREIRA, J. DE L.; CORREAA, B. R. DO P. G.; TORRES, P. L. O uso pedagógico da rede social Facebook. Colabor@-A Revista Digitalda CVA-RICESU, v. 7, n. 28, 2012.

IBGE. IBGE: Metade dos brasileiros teve acesso a internet em 2013. 2014. Disponível em: <http://www.brasil.gov.br/infraestrutura/2014/09/ibge-metade-dos-brasileiros-teve-acesso-a-internet-em-2013>. Acesso em: 21 maio 2016.

JONES, V.; JO, J.; MARTIN, P. Future Schools and How Technology can be used to support Millennial and Generation-Z Students. ICUT (Proceedings B). Anais... 2007. Disponível em: <http://www98.griffith.edu.au/dspace/handle/10072/19022>. Acesso em: 08 set. 2016

MASETTO, M. Inovação na Educação Superior. Interface - Comunicação, Saúde, Educação, v. 8, n. 14, p. 197-202, fev. 2004.

MCCRINDLE, M. The ABC of XYZ: Understanding the Global Generations. 2014. Disponível em: $<$ http://mccrindle.com.au/resources/The-ABC-of-XYZ_Chapter-1.pdf $>$. Acesso em: 12 mar. 2016.

MITRE, S. M. et al. Metodologias ativas de ensino-aprendizagem na formação profissional em saúde: debates atuais. Ciência \& Saúde Coletiva, v. 13, p. 2133-2144, dez. 2008.

MORÁN, J. Mudando a educação com metodologias ativas. Coleção Mídias Contemporâneas. Convergências Midiáticas, Educação e Cidadania: aproximações jovens, v. 2, p. 15-33, 2015. 
MORAN, M.; SEAMAN, J.; TINTI-KANE, H. Teaching, Learning, and Sharing: How Today's Higher Education Faculty Use Social Media. Babson Survey Research Group, 31 mar. 2011.

MOREIRA, J. A.; MONTEIRO, A. M. R. Formação e ferramentas colaborativas para a docência na web social. Revista Diálogo Educacional, v. 15, n. 613, p. 379, 2015.

PORTAL BRASIL. Em 2014, 58,5\% dos estudantes de 18 e 24 anos estavam na faculdade. Portal Brasil. Disponível em: <http:/www.brasil.gov.br/educacao/2015/12/ numero-de-estudantes-universitarios-cresce-25-em-10-anos>. Acesso em: 08 set. 2016.

SCHLEMMER, E. Mídia social em contexto de hibridismo e multimodalidade: o percurso da experiência na formação de mestres e doutores. Revista Diálogo Educacional, v. 15 , n. 45 , p. 399-421, 2015.

SOUZA, A.; SCHNEIDER, H. Aprendizagem Nas Redes Sociais: Colaboração Online Na Prática De Ensino Presencial. SIED: EnPED-Simpósio Internacional de Educaçao a Distância, p. 1-10, 2012.

THE STATISTICS PORTAL. Number of monthly active Facebook users worldwide as of 1st quarter 2016 (in millions). Disponível em: <http://www.statista.com/statistics/264810/ number-of-monthly-active-facebook-users-worldwide/>. Acesso em: 22 maio 2016 a.

THE STATISTICS PORTAL. Number of Facebook users in Brazil from 2014 to 2019 (in millions). Disponível em: <http://www.statista.com/statistics/244936/number-of-facebook-users-in-brazil/>. Acesso em: 22 maio 2016 b.

YOUTUBE. Statistics. Disponível em: <https:/www.youtube.com/yt/press/en-GB/ statistics.html>. Acesso em: 22 maio 2016.

Texto recebido em 03 de janeiro de 2017. Texto aprovado em 09 de fevereiro de 2017. 
